\title{
Water film squeezed between oil and solid: Drainage towards stabi- lization by disjoining pressure
}

\author{
Laure Bluteau, ${ }^{a b c d}$ Maurice Bourrel, ${ }^{c d}$ Nicolas Passade-Boupat, ${ }^{c d}$ Laurence Talini, ${ }^{a b d}$ Emilie \\ Verneuil, ${ }^{a b d}$ and François Lequeux ${ }^{* a b d}$
}

\author{
First published on the web 17th January 2017
}

DOI: 10.1039/c6sm02423h

\begin{abstract}
The spontaneous drainage of aqueous solutions of salt squeezed between an oil drop and a glass surface is studied experimentally. The thickness profile of the film is measured in space and time by reflection interference microscopy. As the film thins down, three regimes are identified: a capillary dominated regime, a mixed capillary and disjoining pressure regime, and a disjoining pressure dominated regime. These regimes are modeled within the lubrication approximation, and the role of the disjoining pressure is precisely investigated in the limit of thicknesses smaller than the range of electrostatic interactions. We derive simple analytical laws describing the drainage dynamics, thus providing tools to uncouple the effect of the film geometry from the effects of the disjoining or capillary pressures.
\end{abstract}

\section{Introduction}

The drainage of a liquid film between two bodies coming into contact, at least one of them being deformable, is a long standing problem; it has been widely addressed in the past decades (see Chan et al. ${ }^{1}$ for a review) and is relevant to many practical applications. For instance, the situation of a liquid drop coming close to a solid surface is encountered in oil recovery processes, whose efficiency in particular depends on the dynamics of the liquid film in between. From a fundamental point of view, it is well known that when a drop or a bubble moves toward a solid surface in a surrounding liquid, it loses its spherical shape. A situation commonly encountered corresponds to a sandwiched liquid film becoming thicker at its center, forming a so-called dimple ${ }^{2-4}$ as a result of the spatial variations of the hydrodynamic pressure. As the liquid film further drains, different effects are at stake, namely viscous dissipation and curvature-induced capillary forces as well as charge-induced and intermolecular interactions for thin films. The full description of drainage accounting for all these effects in a general case requires numerical computations. ${ }^{3,4}$ Most theoretical and experimental studies actually focused on films of thicknesses limited to a given range, in order to neglect either the capillary ${ }^{5,6}$ or the disjoining (including both

a Laboratoire Sciences et Ingénierie de la Matière Molle, CNRS UMR7615, ESPCI Paris, PSL Research University, 10 rue Vauquelin, F-75231 Paris, France, E-mail: francois.lequeux@espci.fr

${ }^{b}$ Laboratoire Sciences et Ingénierie de la Matière Molle, Université Pierre et Marie Curie, Sorbonne-Universités, 10 rue Vauquelin, Paris, France

${ }^{c}$ TOTAL CSTJF, Avenue Larribau, 64000, Pau, France

${ }^{d}$ Laboratoire Physico-chimie des Interfaces Complexes, ESPCI Paris, 10 rue Vauquelin, F-75231 Paris, Bâtiment CHEMSTARTUP, Route Départementale 817, 64170 Lacq, France charge induced and intermolecular interactions) $)^{3,7}$ pressure effects. Even though some questions remain - in particular on the boundary condition at the liquid/liquid interface -, the main features of the mechanism of film thinning are now well established; ${ }^{1}$ however, in the experimental data it remains difficult to separate the effects of the different interactions at stake with purely geometrical parameters, owing to both their strong coupling and lack of analytical descriptions.

Herein we focus on the drainage of an aqueous film formed between an oil drop and a glass surface, with no added surfactant. The solid surface and drop are both immersed in an aqueous electrolyte, and the drop is initially translated toward the glass surface and further stopped; we study the relaxation of the formed dimple toward equilibrium, i.e. toward a flattened film of uniform thickness. Although it has been much less studied than the case of liquid films between a bubble and a solid surface, ${ }^{8,9}$ the drainage of a film between a solid and a liquid drop has been the object of past studies. ${ }^{2,5,10-14}$ Two systems were considered: first, a mercury drop coming closer to a mica surface in water. ${ }^{5,13,14}$ Controlled electrostatic surface potential of the drop allowed for a detailed study of the charge induced interactions between the drop and the solid surface. The second system studied was an oil drop expanded in an aqueous electrolyte toward a silica surface, within conditions in which the two surfaces were charged. ${ }^{2,10-12}$ In particular, the film drainage while relaxing toward equilibrium was characterized without added surfactant and for variable ionic strengths of the electrolyte. ${ }^{2,12}$ In the latter case, different regimes were observed for which the dominant effects were identified and qualitatively discussed. However, no quantitative analysis of the data with respect to the equations describing the drainage dynamics was provided. 
We shed new light on the dynamics of a similar system by different means: First, the drop and solid surfaces are both strongly charged, such that the capillary and disjoining pressure effects are both expected to play a role during drainage, separately but also concomitantly. Second, based on simple mathematical laws to describe the measured deformed shape of the drop, we have developed a theoretical analysis allowing for the derivation of simple analytical laws to describe the film thinning at the different stages of drainage. As a result, the actions of the different effects at stake can be decoupled. In particular, the effect of the geometry of the dimple, which is set by the experimental initial conditions, can be separated from the effects of the capillary and disjoining pressures.

More precisely, we recast our problem within the theoretical background of liquid film thinning: the drainage of a water film formed between a solid and a liquid/liquid interface can be modeled within the framework of the augmented YoungLaplace equation (Eq. 1): within the lubrication approximation, this equation balances out the capillary pressure due to the curvature $\mathscr{C}$ of the liquid/liquid interface of surface tension $\gamma$, the disjoining pressure $\Pi$ and the viscous shear stress within the water film of viscosity $\eta$ and velocity field $v_{r}(z)$ mostly oriented in the radial direction and varying with $z$.

$$
\frac{\partial}{\partial r}(\gamma \mathscr{C}-\Pi)=\eta \frac{\partial^{2} v_{r}}{\partial z^{2}}
$$

Depending on the relative strength of the capillary term and the disjoining pressure term of the pressure gradient, Eq. 1 delineates three regimes: a capillary regime (i), a disjoining pressure dominated regime (iii), and a mixed capillary/disjoining pressure regime (ii). We show that the capillary regime is observed in the early stages of the drainage while the disjoining pressure regime arises when film thicknesses have decreased enough to lay within the range of the long range interactions. Eventually, if long-range interactions are repulsive and if no dewetting occurs, the film stabilizes at a uniform thickness for which the Laplace pressure in the drop balances the disjoining pressure in the film. A mixed regime (ii) is obtained at mid course, for which the thinnest part of the film, located at the edge, is dominated by the disjoining pressure, while the center of the film is thick enough to be described by the capillary and viscous stresses.

In cylindrical geometry, Eq. 1 can be written as a function of the film thickness and its derivatives. The full derivation is detailed in the Appendix. The thickness profile of the film $h(r, t)$ therefore obeys the following equation:

$$
\frac{\partial P}{\partial r}=\frac{\partial}{\partial r}\left(-\gamma\left(\frac{\partial^{2} h}{\partial r^{2}}+\frac{1}{r} \frac{\partial h}{\partial r}\right)-\Pi(h)\right)=\frac{-12 q \eta}{(3 \beta-2)} \frac{1}{h^{3}}
$$

where $2 \pi r q(r, t)$ is the local outward flow and is related to the time derivative of $h$ by the mass conservation equation:

$$
\frac{\partial r q}{\partial r}=-r \frac{\partial h}{\partial t}
$$

The parameter $\beta$ sets the boundary condition at the liquid/liquid interface: for a no-slip condition, $\beta=1$, and for a zero tangential stress condition, $\beta=2$.

By performing a simple analysis of the augmented YoungLaplace equation together with mass conservation, we show in this paper that our experimental results on the spontaneous drainage of squeezed water films between oil and solid can be described through simple analytical laws of the time dependence of the thickness profile which can be separated into three dynamic regimes. We propose a simple method to measure the oil/water interface boundary condition and the disjoining pressure as a function of the thickness.

\section{Experimental Section}

In this paper, we focus on the spontaneous drainage of a water or brine film squeezed between an oil drop and a glass substrate. We chose to approach the oil drop of controlled size immersed in a water or brine bath towards a glass substrate at a constant velocity. At a given distance to the substrate, the drop is stopped and we measure the subsequent characteristics of the spontaneous drainage of the water or brine squeezed film.

The experimental setup is schematically depicted in Fig. 1 and is similar to setups existing in the literature. ${ }^{1,2,9,10,15,16}$ The water bath is filled with a solution of $\mathrm{NaCl}$ (Normapur - purity $\geqslant 99.5 \%$ ) in deionized water (resistivity $2.1 \mathrm{M} \Omega . \mathrm{cm}$ at $23.1^{\circ} \mathrm{C}-\mathrm{pH}=5$ ). An oil drop of dodecane (Sigma Aldrich - purity $\geqslant 99 \%$ ) is formed within the reservoir at the end of a capillary tube made of inert polymer, of inner diameter $65 \mu \mathrm{m}$ and outer diameter $1.59 \mathrm{~mm}$. Fig. 1a) shows a side view of the drop. Once the drop filled, a waiting time of 300 seconds was chosen in order to reach an equilibrium at the oil water interface. Indeed, this time corresponds to the characteristic time of decay of the dodecane/water surface tension measured by the pendant drop method (by around $8 \%$ ). The dodecane/water interfacial tension stabilizes at $\gamma=42.5 \pm 0.5 \mathrm{mN} / \mathrm{m}$ for the next 10 minutes, which is far larger than the experimental time. This value of the interfacial tension does not depend on the $\mathrm{NaCl}$ concentration.

As glass substrate, we used plano-convex borosilicate lenses with radius of curvature of $233.4 \mathrm{~mm}$. This borosilicate glass has an iso-electric point around 3.6. ${ }^{17}$ As a consequence, in deionized water at $\mathrm{pH} 5$, the glass/water interface presents a negative charge: the silanol groups $\mathrm{SiOH}$ are partially ionized into $\mathrm{SiO}^{-}$at the interface. The glass lens is mounted on a microscope objective $(10 \mathrm{x}, \mathrm{NA}=0.3)$. 


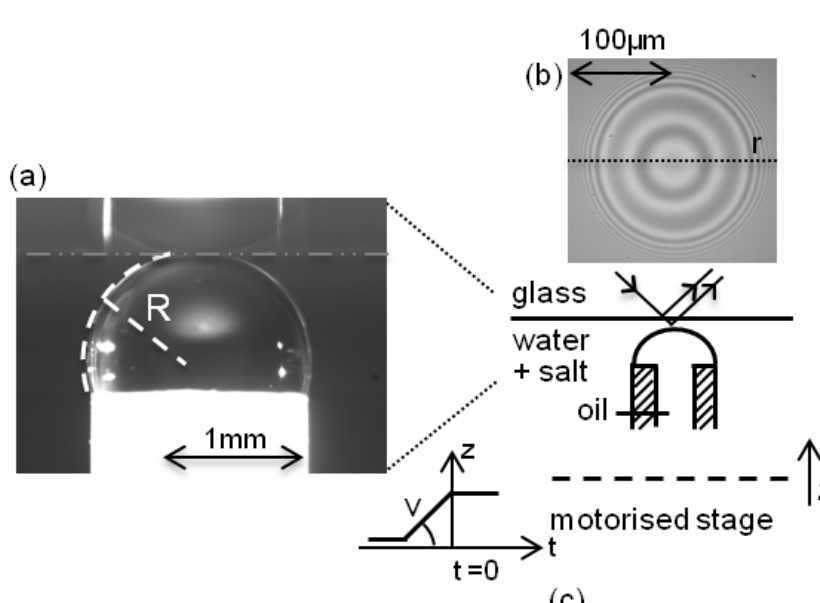

(c)

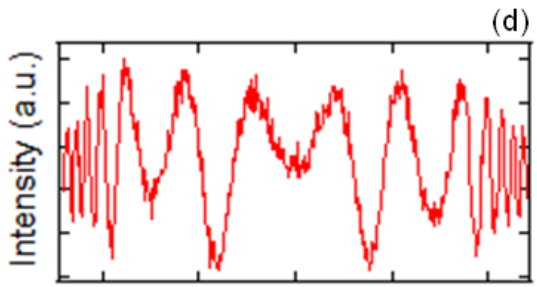

(e)

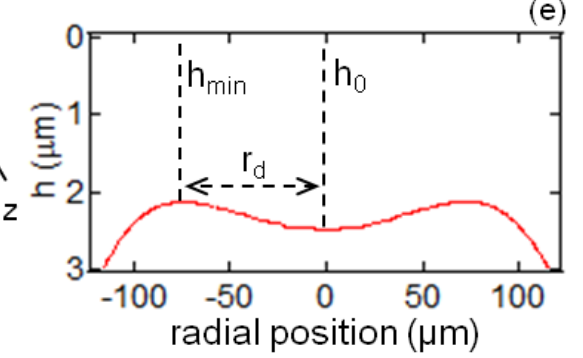

Fig. 1 Experimental setup. (a) Side view of a dodecane drop immersed in water/ $\mathrm{NaCl}$ solution squeezed against a glass substrate with radius of curvature R. (b) Top view of the squeezed film by reflection interference microscopy: monochromatic illumination through a $\mathrm{x} 10$ objective and a 44 magnification glass collected by reflection and forms equal thickness fringes between the oil/water and the water/glass interfaces. (c) The oil drop is formed at the end of an inert polymer tube and placed in the optical axis of the objective, the drop is raised at constant velocity $\mathrm{v}$, then stopped at time $t=0$. (d) Profile of the interference intensity along a diameter of the squeezed film and absolute thickness profile of the water film. The geometry of the water film is characterized by its thickness at the center $h_{0}$, its thinnest thickness $h_{m i n}$ and its radius $r_{d}$.

To control the relative position of the drop and the glass substrate, the tube is attached to the water tank, which is set to a vertical motorized stage (Fig. 1c). Once at equilibrium with water, the oil drop is driven toward the solid surface at a constant velocity ranging between 0.1 and $20 \mathrm{~mm} / \mathrm{s}$, and then stopped. The approach step results into the creation of a squeezed water film with a structure called dimple: ${ }^{3}$ the film is thinner at the edge. The dimple is characterized by three lengths: the thickness at the center $h_{0}$, the thickness at the barrier ring $h_{\min }$, and the radial distance between those two thicknesses, the dimple radius $r_{d}$ (Fig. 1e). The dimple profile is measured over time by an interferometry technique: The drop is placed in the optical axis of the microscope objective and observation is made through the glass substrate, using a magnifying lens $(4 \mathrm{x})$. Episcopic monochromatic illumination $(\lambda=615 \mathrm{~nm})$ is provided by a LED and optical filters. Images are collected with a fast camera at a speed ranging between 50 and 1000 frames per second for 10 to $40 \mathrm{~s}$. The squeezed film thickness being in the micrometer range, fringes of equal thickness form due to interferences between reflected light at the oil/water and water/glass interfaces, as shown in the image of Fig. 1b). A typical intensity profile along the dimple diameter is presented in Fig. 1d). Two successive intensity minima correspond to a relative thickness variation of $\pm \lambda / 2 n$ where $n$ is the water or brine refractive index. An absolute reference is obtained from the homogeneous equilibrium thickness $h_{e q}$ at the end of the film drainage by alternatively using three different wavelengths $(\lambda=615 \mathrm{~nm}$, $530 \mathrm{~nm}, 445 \mathrm{~nm}$ ) in the microscope illuminator. In some cases, the oil wets the glass substrate thus providing a zero thickness reference. To monitor the pressure into the oil drop $P_{\text {drop }}$, a side view is captured by a CCD camera as shown in Fig. 1a) from which the radius of curvature $R$ of the drop is measured. The pressure is computed using the Laplace equation $P_{\text {drop }}=P_{\text {ext }}+2 \gamma R^{-1}$ where $\gamma$ is the oil/brine interfacial tension and $P_{\text {ext }}$ is the reservoir pressure. We performed series of experiments consisting in approaching drops with radii of curvature ranging between 0.66 and $1.43 \mathrm{~mm}$ to change the Laplace pressure in the drop.

At the beginning of the spontaneous drainage of the dimple, defined as initial time $t=0$, the characteristic lengths of the dimple depend on the drop approach velocity and on the final distance between the capillary tube and the glass substrate. The faster the approach step, the thicker the dimple, the larger $h_{0}$. The shorter the distance between the capillary tube end and the glass, the wider the dimple, the larger $r_{d}$. We performed series of experiments with varied approach velocity and tube/glass distance. We obtained dimple radii $r_{d}$ ranging between 60 to $130 \mu \mathrm{m}$, and initial dimple thicknesses $h_{0}$ ranging between 1.4 and $2.4 \mu \mathrm{m}$.

Finally, the electrostatic interactions were controlled by using $\mathrm{NaCl}$ aqueous solutions with concentrations ranging be- 
tween $10^{-6}$ and $10^{-1}$ mol.L $\mathrm{L}^{-1}$. Away from a charged surface in aqueous solution, the range of the electrostatic interactions is set by the Debye length $\lambda_{D}$ given by:

$$
\lambda_{D}=\sqrt{\frac{\varepsilon_{r} \varepsilon_{0} k_{B} T}{\sum n_{j} q_{j}^{2}}}
$$

with $\varepsilon_{r}$ the relative permittivity, $\varepsilon_{0}$ the permittivity of vacuum, $k_{B}$ the Boltzmann constant, $T$ the temperature, $n_{j}$ the density of ionic species $\mathrm{j}$ of charge valence $q_{j}$. To estimate $\lambda_{D}$ the species accounted for were $\mathrm{Na}^{+}, \mathrm{Cl}^{-}, \mathrm{H}_{3} \mathrm{O}^{+}, \mathrm{OH}^{-}$and $\mathrm{HCO}_{3}^{-}$.

\section{Film thinning}

The drainage dynamics is studied through the time evolution of the film thickness at the center $h_{0}$ and at the edge $h_{m i n}$, also called the barrier ring. A typical example is shown in Fig. 2a). Similar measurements were reported in the literature. $1,2,10,12$ At the barrier ring, the thickness is lesser and its thinning rate is larger that at the center. As a consequence the thickness at the barrier ring is first to reach its equilibrium thickness. Eventually, the film comes to an equilibrium state with a uniform thickness denoted $h_{e q}$. In Fig. 2b), a log-log plot of the time variation of the two thicknesses in the early stage of the drainage evidences a power law dependence of both $h_{0}$ and $h_{\text {min }}$ with time. We denote $t_{1}$ and $h_{1}$ the time and thickness at the barrier ring when the measured thickness departs from this first power law regime. At later times, the thickness difference $h_{0}-h_{\min }$ decays exponentially over time: in Fig. 2c) where $\ln \left(\frac{h_{0}-h_{\min }}{h_{\min }}\right)$ was plotted versus time, we observe two exponentially decaying laws with two successive characteristic times. We denote $t_{2}$ and $h_{2}$ the time and thickness at the center for which the crossover between these last two regimes is measured. Altogether, the drainage dynamics can be separated into three different regimes, the crossovers being determined when, first, the barrier ring $\left(h_{\text {min }}\right)$ reaches a crossover value $h_{1}$ and second, the center of the dimple $\left(h_{0}\right)$ reaches the crossover value $h_{2}$. The values of the cross-over thicknesses are plotted against the Debye length in Fig. 3 for a series of experiments with drops of similar radius of curvature.

\subsection{Equilibrium thickness and disjoining pressure}

The equilibrium thickness $h_{e q}$ results from an equilibrium between long range repulsive electrostatic interactions and the overpressure of the drop. As a consequence, this thickness depends strongly on the Debye length (Fig. 3): the higher the concentration, the thinner the equilibrium thickness. ${ }^{2}$ The equilibrium thickness may be large compared to the Debye length. This is due to the very low overpressure applied on the film, around $100 \mathrm{~Pa}$.

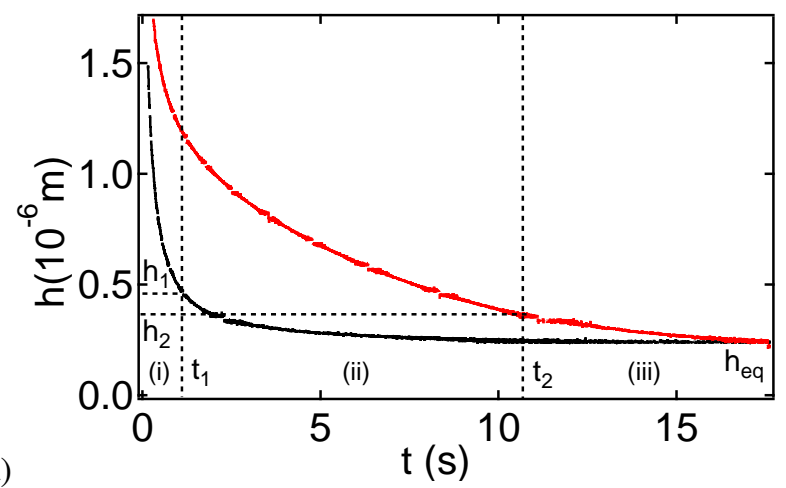

(a)

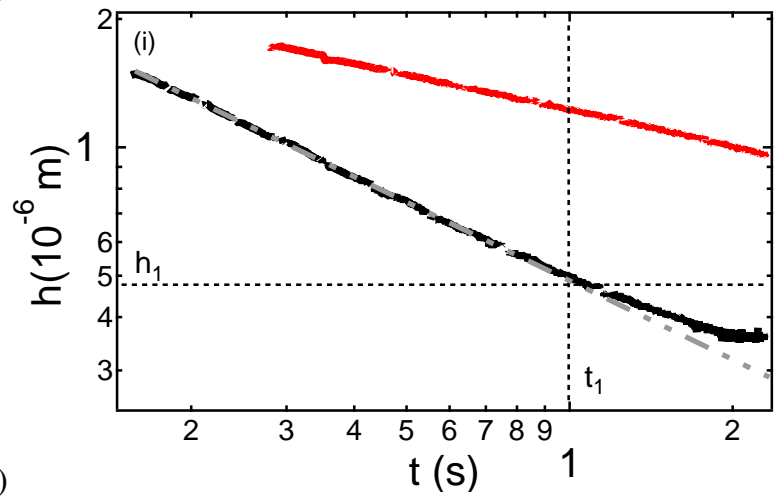

(b)

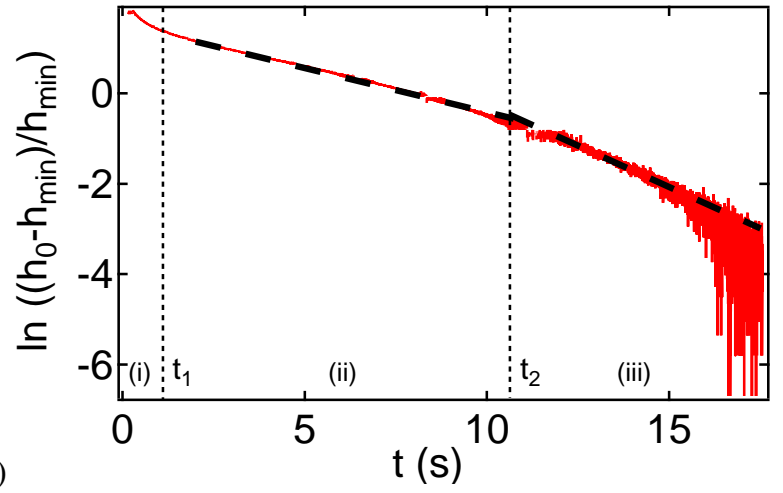

Fig. 2 (a,b) Time evolution of $h_{0}$ (red/light curve) and $h_{\min }$ (black/dark curve) for a $\mathrm{NaCl}$ solution of $10^{-6} \mathrm{M}\left(\lambda_{D}=166 \mathrm{~nm}\right)$. (a) Linear plot over the whole drainage. (b) Log-log plot of the first drainage regime. Time $t_{1}$ and thickness $h_{1}$ both mark the end of regime (i) on the $h_{\min }(t)$ curve. (c) Time variation of $\ln \left[\left(h_{0}-h_{\min }\right) / h_{\min }\right]$ over the whole drainage. Dashed lines delineate regimes (ii) and (iii). Time $t_{2}$ and corresponding thickness $h_{2}$ of $h_{0}$ define the crossover between regime (ii) and (iii). Initial thickness at the dimple center is $h_{0}(0)=1.6 \mu \mathrm{m}$, drop radius of curvature $R=0.8 \mathrm{~mm}$. 


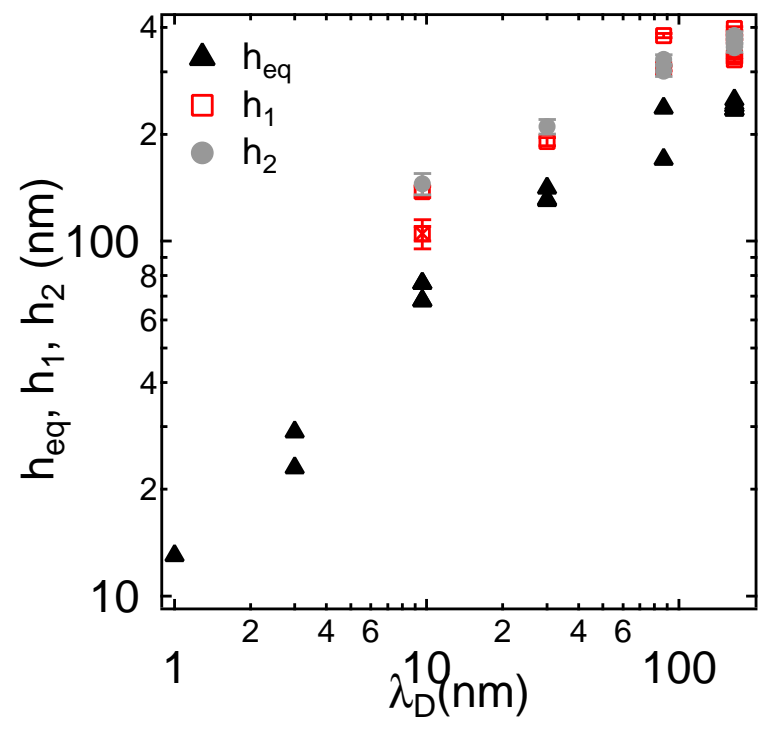

Fig. 3 Equilibrium thickness $h_{e q}$ (triangle) and crossover thicknesses $h_{1}$ (square) and $h_{2}$ (circle) between regimes (i) and (ii) and between regimes (ii) and (iii) respectively, as a function of Debye length $\lambda_{D}$ for $\mathrm{NaCl}$ solutions of concentration ranging between $10^{-6}$ and $10^{-1} \mathrm{M}$. Drop radius of curvature $R=0.8 \pm 0.05 \mathrm{~mm}$

The long range electrostatic interactions are described through the disjoining pressure $\Pi$ in the brine film defined as the derivative of the interaction potential with respect to film thickness $h$.

Let us compare the pressure in the film and in the drop: in the drop, the overpressure compared to the reservoir writes: $P_{\text {drop }}-P_{\text {ext }}=2 \gamma R^{-1}$. In the brine film of curvature $\mathscr{C}$, the overpressure across the oil/brine interface writes $\gamma \mathscr{C}$ and finally, the pressure in the film writes:

$$
P=P_{\text {ext }}+2 \gamma R^{-1}+\gamma \mathscr{C}-\Pi
$$

At equilibrium, when the film reaches a uniform thickness $h_{e q}$, the film curvature $\mathscr{C}$ is uniformly zero and the film pressure equilibrates with the external pressure $P_{\text {ext }}$. Altogether, the disjoining pressure balances the Laplace pressure in the drop: $\Pi\left(h=h_{e q}\right)=2 \gamma R^{-1}$. Therefore, from the measurements of the equilibrium thickness and the drop radius of curvature, we can measure the variations of the disjoining pressure $\Pi$ with thickness $h$ as done in other studies. ${ }^{5,18}$ The results are reported in Fig. 4 for different salt concentrations of the brine. Measurements of $\Pi(h)$ found in the literature are abundant for films squeezed between bubbles or drops in emulsions, ${ }^{19,20}$ they are however scarce in liquid/liquid/solid systems as reported here. Next we compare our measurements of disjoining pressure with charge induced interactions.

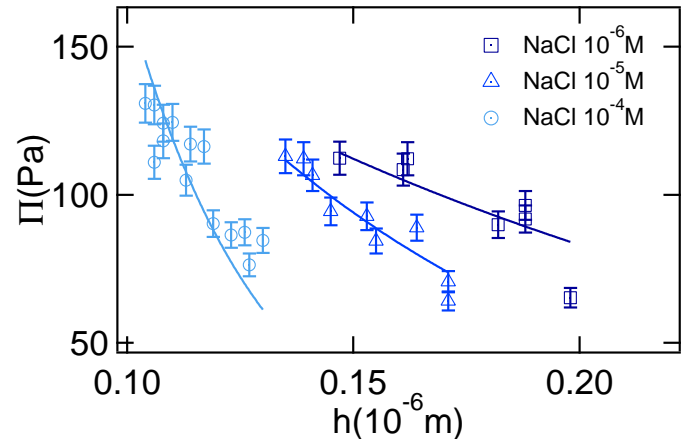

Fig. 4 Disjoining pressure $\Pi$ as a function of brine film thickness $h$ for $\mathrm{NaCl}$ solutions with concentration $10^{-6} \mathrm{M}, 10^{-5} \mathrm{M}, 10^{-4} \mathrm{M}$ from lighter to darker blue, measured on homogeneous films at equilibrium obtained with drops of radius of curvature $R$ varying between 0.6 and $1.4 \mathrm{~mm}$. Markers - experimental data. Lines - fit to Eq. 6 for an oil/water surface potential $\psi_{o w}$ of $-66 \mathrm{mV}$, and glass/brine interfacial potential $\psi$ as a fitting parameter.

It has been known for long that oil drops in water can bear charges according to the $\mathrm{pH}$ value. ${ }^{21,22}$ In a recent study, it was demonstrated that these charges result from the presence at the oil/water interface of fatty acids, that are part of the impurities found in commercial oils. ${ }^{23}$ For large enough $\mathrm{pH}$ values, the carboxyl groups of the fatty acids ionize and the oil drops present a surface potential that can reach tens of millivolts. In agreement with those findings, charges on the drop surface are needed in order to account for the disjoining pressures we measure. Actually, considering only the charge of the glass surface yields surface potentials close to $1 \mathrm{~V}$, which is far larger than the one expected for glass. ${ }^{24}$ There is no exact analytical solutions of the non linear Poisson Boltzmann equation for two asymmetrically charged surfaces, but an approximate relation is found in the literature in those conditions. ${ }^{25,26}$ In that frame, the variations of the disjoining pressure with aqueous film thickness is written as:

$$
\Pi(h)=\left(64 n_{0} k_{B} T \tanh \left(\frac{e \psi_{o w}}{4 k_{B} T}\right) \tanh \left(\frac{e \psi}{4 k_{B} T}\right)\right) e^{\left(-h / \lambda_{D}\right)}
$$

with $k_{B}$ the Boltzmann constant, $\mathrm{T}$ the temperature, $\lambda_{D}$ the Debye length, $n_{0}$ the number of ions per volume, $e$ the elementary charge, $\psi$ the glass/brine interfacial potential and $\psi_{\text {ow }}$ the oil/water surface potential. To describe the experimental data of $\Pi(h)$ in Fig. 4, the oil/water interface potential was estimated from Roger's paper: ${ }^{23} \psi_{\text {ow }} \sim-66 \mathrm{mV}$ at $[\mathrm{NaCl}]=10^{-5} \mathrm{~mol} \cdot \mathrm{L}^{-1}$. This value is supported by Marinova et al. ${ }^{21}$ The experimental data was fitted to Eq. 6 with the electrostatic potential of glass $\psi$ as a fitting parameter. The 
values we found are in agreement with data from the literature; ${ }^{24}$ for instance, a potential $\psi \sim-21 \mathrm{mV}$ was obtained for a $10^{-5} \mathrm{M} \mathrm{NaCl}$ solution shown in Fig. 4.

(a)

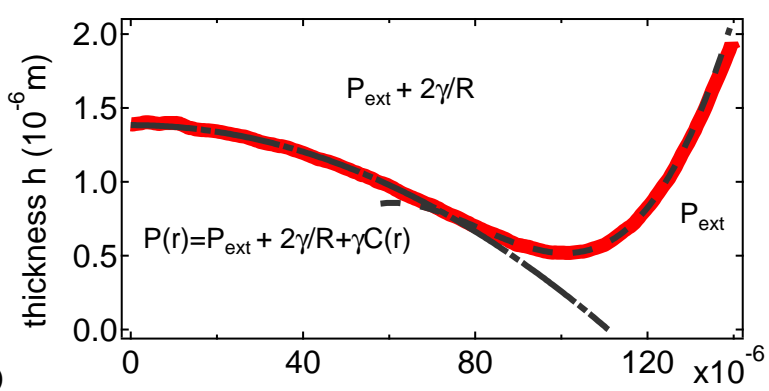

(b)

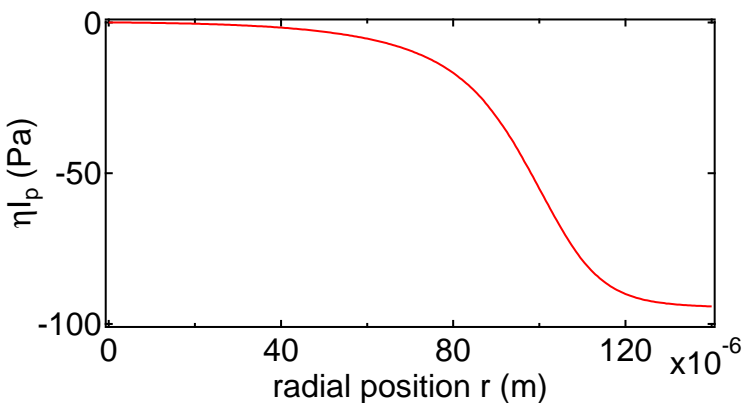

Fig. 5 (a) Thickness profile of a water film $h(r)$ of an aqueous solution of $[\mathrm{NaCl}]=10^{-} 1 \mathrm{M}$ as a function of the radial distance to the center $r$. Dash-dotted line: fit to a second order polynomial at the center. Dashed line: fit to a third order polynomial at the barrier ring. (b) Radial variations of the viscous contribution to the film pressure $\eta I_{p}$ (to a numerical factor) as defined by Eqs. 9 and 10: most of the viscous stress gradient is located at the barrier ring.

\subsection{Pressure in the film}

During the course of drainage, we show next how the space and time variations of the experimental profiles of the dimple thickness (Fig. 5a) can be used to measure the overpressure in the film. On one hand, the pressure at the center of the dimple $P(0)$ can be written using Eq. 5, noting that the oil/brine interface curvature $\mathscr{C}(0)=-2\left(\frac{\partial^{2} h}{\partial r^{2}}\right)_{r=0}$ can be measured from the radial profiles.

$$
P(0)=P_{\text {ext }}+\frac{2 \gamma}{R}+\gamma \mathscr{C}(0)-\Pi\left(h_{0}\right)
$$

On the other hand, the pressure gradient in the film is given by the Stokes equation in the lubrication approximation (Eq. 2) as a function of the flow rate $q$. First, $q$ can be computed from the experimental profiles using the mass conservation equation (Eq. 3) and second, the pressure $P(r)$ can be derived by integration of Eq. 2 between the dimple center and current position $r$, yielding:

$$
\begin{array}{r}
P(r)=P(0)+\frac{\eta}{(3 \beta-2)} I_{p}(r) \\
P(r)=P_{e x t}+\frac{2 \gamma}{R}+\gamma \mathscr{C}(0)-\Pi\left(h_{0}\right)+\frac{\eta}{(3 \beta-2)} I_{p}(r)
\end{array}
$$

where the integral $I_{p}$ writes

$$
I_{p}(r)=12 \int_{0}^{r} \frac{d r^{\prime}}{r^{\prime} h\left(r^{\prime}\right)^{3}} \int_{0}^{r^{\prime}} r^{\prime \prime} \frac{\partial h\left(r^{\prime \prime}\right)}{\partial t} d r^{\prime \prime}
$$

In Eq. 9, the viscous stress writes $\frac{\eta}{(3 \beta-2)} I_{p}$ and $I_{p}$ can be determined experimentally from geometrical measurements only, using the time and space variations of $h(r, t)$. A typical example is reported in Fig. 5b) where $\eta I_{p}$ is plotted as a function of the radial distance to the film center. We observe that most of the pressure drop - and thus the viscous dissipation is located at the dimple edge, at the barrier ring.

Finally, the film curvature $\mathscr{C}$ is measured by fitting the experimental profile $h(r)$ at the center to a second order polynomial. At the barrier ring, a third order polynomial is used to fit the profile (Fig. 5 a).

\section{Three drainage regimes}

In the following, we describe the three regimes identified during the drainage dynamics of the dimple. Our hypothesis is that, in the early times of the drainage for which the thickness of the film is larger that the crossover thickness $h_{1}$, the disjoining pressure can be neglected compared to the capillary pressure. In the typical example of Fig. 2, using our measurements of the disjoining pressure versus thickness at equilibrium, we can check experimentally that, indeed, $\Pi\left(h_{1}\right)$ calculated through the model Eq. 6 is of the order of $15 \mathrm{~Pa}$, therefore smaller than the capillary pressure gradient $\Delta\left(\gamma_{\mathcal{C}}\right)$ measured over the same distance to be of $60 \mathrm{~Pa}$. This defines the capillary regime in which the viscous stress is balanced by the capillary pressure gradient only. When the thickness at the barrier ring becomes smaller than $h_{1}$, the drainage enters a mixed regime where the film pressure in the center of the dimple is dominated by the interfacial tension term, while at the barrier ring, it is controlled by the competition between disjoining pressure and capillary pressure. Finally, in the last regime, both the film curvature and the film thickness have decreased enough so that the film pressure is controlled by the disjoining pressure only, which is balanced out by the viscous stress. This defines the disjoining pressure regime. The crossover between the last two regimes was observed experimentally at a thickness $h_{2}$ of the dimple center (Fig. 2a,c) while the thickness at the barrier ring has already reached its minimum value: $h_{\min } \sim h_{e q}$ : using again 
the disjoining pressure versus thickness curve (Fig. 4), we compute the corresponding difference of disjoining pressure: $\Pi\left(h_{e q}\right)-\Pi\left(h_{2}\right) \sim 25 \mathrm{~Pa}$ while the capillary pressure difference is of $15 \mathrm{~Pa}$. We hence verify that the crossover between regimes (ii) and (iii) is obtained when the capillary term in the pressure gradient vanishes compared to the disjoining pressure term.

\subsection{Capillary regime}

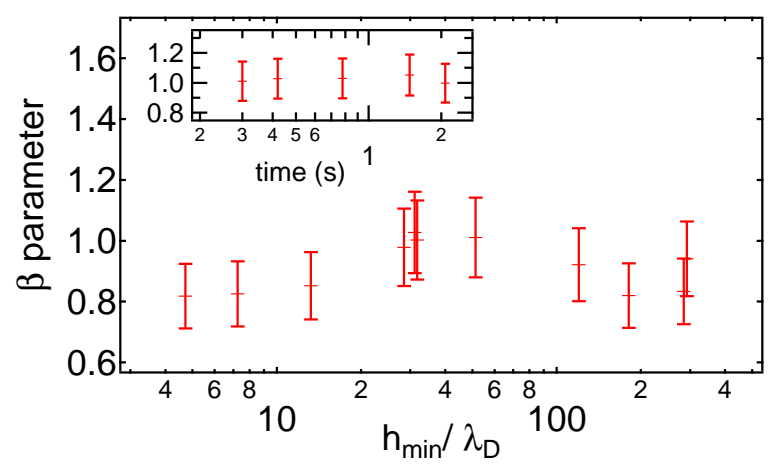

Fig. 6 (insert) - Time variation of the boundary condition parameter $\beta(t)$ over the capillary regime (i) of a drainage experiment with $[\mathrm{NaCl}]=10^{-5} \mathrm{M}$ and drop radius $R=0.78 \mathrm{~mm}$ : no time dependence of $\beta$ observed. (main) Boundary condition parameter $\beta$ as a function of the current minimum thickness $h_{\min }$ at the barrier ring normalised by the Debye length $\lambda_{D}$, for $\mathrm{NaCl}$ concentrations varying between $10^{-6} \mathrm{M}$ and $10^{-1} \mathrm{M}$. Data are collected at mid-course of regime (i) (time $t=t_{1} / 2$ ).

In this regime, the disjoining pressure can be neglected compared to the capillary pressure and $\Pi\left(h_{0}\right) \sim 0$. Eq. 9 can then be evaluated for $r=\infty$, at which $P=P_{\text {ext }}$, yielding:

$$
0=\frac{2 \gamma}{R}+\gamma \mathscr{C}(0)+\frac{\eta}{(3 \beta-2)} I_{p}(\infty)
$$

Rearranging the latter equation, an expression for the boundary condition parameter $\beta$ can be obtained:

$$
\beta=\frac{1}{3}\left(2-\frac{\eta I_{p}(\infty)}{2 \gamma / R+\gamma \mathscr{C}(0)}\right)
$$

The parameter $\beta$ was deduced from the measurements of the space and time dependent profiles $h(r, t)$, the viscosity $\eta$, and the interfacial tension $\gamma$ using Eq. 12. As shown in the insert of Fig. 6 , the boundary condition parameter $\beta$ was found to be constant over time in the capillary regime. Figure 6 shows the $\beta$ values for all experiments: interestingly, its average value is $\beta=0.9 \pm 0.1$, that is, a value smaller than 1 . The case $\beta=1$ would correspond to a no-slip or tangentially immobile boundary condition, while a liquid/liquid interface with no shear stress at the interface is described by $\beta=2$. Therefore, not only the expected liquid/liquid interface $(\beta=2)$ boundary condition is not obtained, but the solid/liquid boundary condition does not apply neither. In the literature, the tangentially immobile boundary condition $(\beta=1)$ was found to apply to different fluid interfaces such as gas bubble/water ${ }^{1,27}$ or mercury/water. ${ }^{5,28}$ No reports of $\beta$ values smaller than 1 can be found, to the best of our knowledge. However, values of $\beta$ were not directly measured before, instead, theoretical numerical solutions computed for $\beta=1$ or $\beta=2$ were compared to the experimental data, ${ }^{15,29}$ and no values smaller than 1 were ever considered. In the following, we offer a possible explanation to account for our result. A boundary condition parameter $\beta$ smaller than 1 corresponds to a velocity at the oil/water interface directed inward, towards the center of the dimple. Both our measurements of the oil/water interfacial tension (Section 2) and the oil/water surface potential (Section 3.1) show the adsorption of traces of contaminants at this interface. When surface-active molecules (either added surfactants or contaminants) are present, even in small quantities, they accumulate at the interface and Marangoni stresses may develop, rendering the interface tangentially immobile, as observed in the literature, where a variation of interfacial tension as small as $0.1 \mathrm{mN} . \mathrm{m}^{-1}$ along an interface is sufficient to explain the tangentially immobile boundary condition. ${ }^{27,29}$ In our case, an estimate of the interfacial tension variations resulting in a no-slip boundary condition is provided by balancing the Marangoni stress $\partial \gamma / \partial r \sim \Delta \gamma / \delta r$ with the viscous tangential stress at the interface, where $\delta r$ is the radial width over which the dissipation occurs. It can be estimated from the Fig. $5 \mathrm{~b}(\delta r \sim 20 \mu m)$. It yields: $\Delta \gamma / \delta r \sim \frac{3}{2} \frac{\partial h_{0}}{\partial t} \frac{\eta \delta r}{h_{\min }^{2}}$ and to an amplitude of the difference of interfacial tension of $\Delta \gamma \sim 0.3 \mathrm{mN} \cdot \mathrm{m}^{-1}$. This value is ten times smaller than the decrease over time of the oil/water interfacial tension measured by the tensiometer, validating the onset of Marangoni stresses at the oil/water interface. Now, recalling that as the droplet is driven towards the glass surface, the water flow may drive the surface-active molecules outward, the drop apex may be initially depleted in surfactants, creating an interfacial tension gradient before the drainage step itself starts. For such a Marangoni stress to hold, the timescale for the surface-active molecules to equilibrate with the bulk oil must be large compared to the total duration of the drop approach and of the capillary regime. The latter is of the order of 2 seconds, while the former is taken from the tensiometry experiments where the oil/water surface tension decrease is monitored over time and found to be of 300 seconds. Therefore, the surface tension gradient created during the approach phase probably doesn't have time to relax. During the drainage, once the drop is stopped, this pre-existing 
interfacial stress generates a counter-flow at the liquid/liquid interface, directed from the outside to the center of the dimple, consistent with a boundary condition value smaller than 1 .

When the interfaces are charged, as considered herein, charge-induced interfacial effects, namely electro-osmotic effects, ${ }^{30}$ could also be expected to contribute to the boundary condition. The resulting value of $\beta$ would in that case depend on the ionic strength of the electrolyte and more precisely on the ratio of the thickness at the barrier ring to the Debye length, $h_{\min } / \lambda_{D}$. We report in Fig. 6 the variations of the parameter $\beta$ at midcourse of regime (i) (time $t=t_{1} / 2$ ) for salt concentrations ranging from $10^{-6}$ to $10^{-1} \mathrm{M}$ and as a function of $h_{\min } / \lambda_{D}$. No significant variations of the parameter $\beta$ is observed although $h_{\min } / \lambda_{D}$ spans more than two decades. Therefore, we conclude that the boundary condition we measure at the oil/water interface does not result from an electro-osmotic effect, but from Marangoni stresses induced by the presence of surface-active contaminants and built-up during the drop approach step.

Special care to keep the surfaces clean and use of purified dodecane would modify the boundary condition. Using a 2D ideal gas equation to relate the difference in interfacial tension estimated above, $\Delta \gamma \sim 0.3 \mathrm{mN} . \mathrm{m}^{-1}$, to the surface concentration of contaminants $\Gamma: \triangle \gamma=k T \Gamma$, the estimated fraction of contaminant molecules in oil should then be smaller than approximately $10^{-5} \%$ to avoid this Marangoni effect, which would require the use of extremely pure oil together with very clean surfaces and water. These conditions cannot be fulfilled easily, as far as we know.

In Fig. 7a,b), we report the time variations of $h_{0}$ and $h_{\min }$ in the capillary regime (i). Models from the literature, ${ }^{3,7,25}$ as our own analysis developed in the Appendix, predict a power law dependence with time of both the center thickness $h_{0}$ and the barrier ring thickness $h_{\min }$. Our own analysis provides the following power laws, in the case of $\beta=1$ :

$$
\begin{gathered}
h_{0}(t) \simeq \frac{3}{4}\left(\frac{\eta r_{d}^{6}}{\gamma R}\right)^{1 / 4} t^{-1 / 4} \\
h_{\min }(t) \simeq \frac{3}{4} \sqrt{\frac{\eta R r_{d}^{2}}{\gamma}} t^{-1 / 2}
\end{gathered}
$$

Either from the literature or from our own derivation, the predicted exponent is $\mu=1 / 4$ for the center thickness and $v=1 / 2$ for the barrier ring thickness. In Fig. 7a,b), our experimental data have been fitted to the following power laws: $h_{0}(t)=A_{0} t^{-\mu}$ and $h_{\min }(t)=A_{\min } t^{-v}$. We find $\mu=0.26 \pm 0.03$ and $v=0.55 \pm 0.04$ in good agreement with the predictions for the exponents. More controversial are the numerical prefactors for the power laws: different expressions were found in the past, depending on the approximation methods used. Here, based on our independent measure of the parameter $\beta$ we choose to set $\beta$ to 1 . In Fig. 7c) and d), we plot the measured prefactors $A_{0}$ and $A_{\min }$ as a function of their theoretical expression $\left(\frac{\eta r_{d}^{6}}{\gamma R}\right)^{1 / 4}$ and $\sqrt{\frac{\eta R r_{d}^{2}}{\gamma}}$, where the dimple radius $r_{d}$ and the drop radius $R$ were measured experimentally. The data were fitted to a line and we experimentally find:

$$
\begin{gathered}
A_{0}=(0.52 \pm 0.02)\left(\frac{\eta r_{d}^{6}}{\gamma R}\right)^{1 / 4} \\
A_{\text {min }}=(1.06 \pm 0.07) \sqrt{\frac{\eta R r_{d}^{2}}{\gamma}}
\end{gathered}
$$

The numerical prefactors we predict are 0.75 for both $h_{0}$ and $h_{\min }$, while those predicted by Hartland et al. ${ }^{7}$ are 0.17 and 0.74 , respectively, and those predicted by Frankel and Mysels $^{3}$ are 0.44 and 0.49 respectively: they all lay within the same range of magnitude. Our experimental determination of the drainage dynamics in the capillary regime (i) is therefore in good agreement with both our model and models from the literature. This confirms the relevance of our approach - as described in Appendix.

As the film is thinning, the thickness at the barrier ring comes into the range of electrostatic interactions. As mentioned earlier, as the disjoining pressure gradient becomes of the same order of magnitude as the capillary pressure gradient, both terms are to be considered in the augmented Young Laplace equation (Eq. 1). In the following, we discuss the drainage dynamics within this mixed regime.

\subsection{Mixed regime}

The drainage dynamics in the mixed regime (ii) was described within the following approximations, as detailed in the Appendix: the thickness at the barrier ring is close to its equilibrium value $h_{e q}$, and thus set by a balance between the disjoining pressure and the overpressure in the drop. At the dimple center, the thickness is large enough for the disjoining pressure to be vanishing and the oil/brine interface curvature $\mathscr{C}$ is small compared to the overpressure $2 \gamma R^{-1}$ in the drop. Altogether, the pressure gradient over the dimple radius, that drives the flow outwards, can be derived from Eq. 9:

$$
P(0)-P(\infty)=P(0)-P_{e x t} \sim \frac{2 \gamma}{R}
$$

The viscous stress is more difficult to estimate. However, simple geometrical arguments on the shape of the barrier ring 


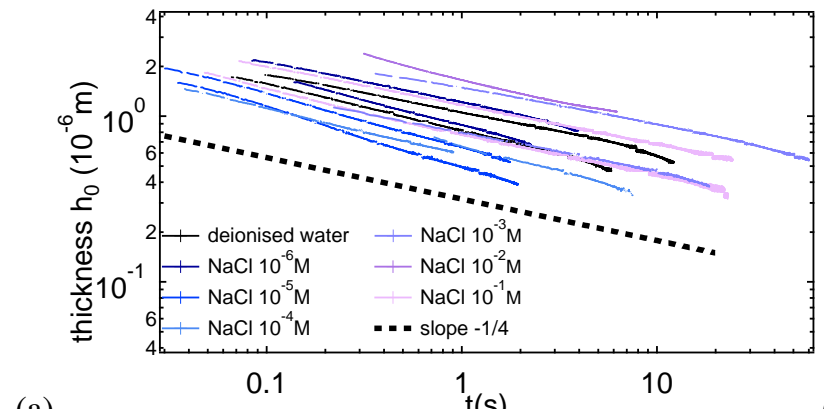

(a)

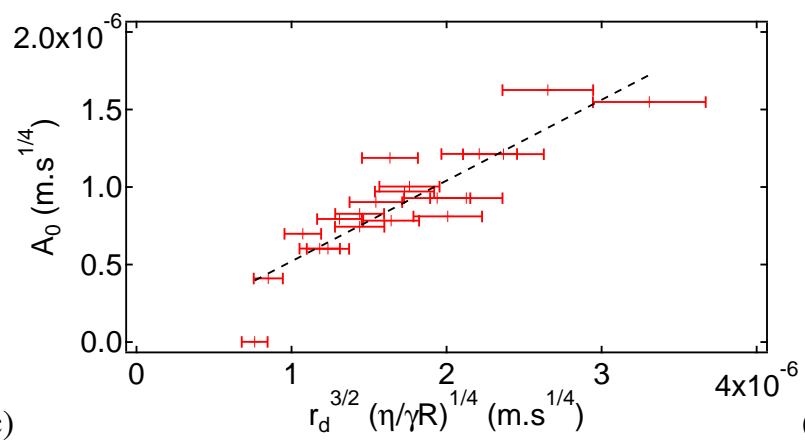

(b)
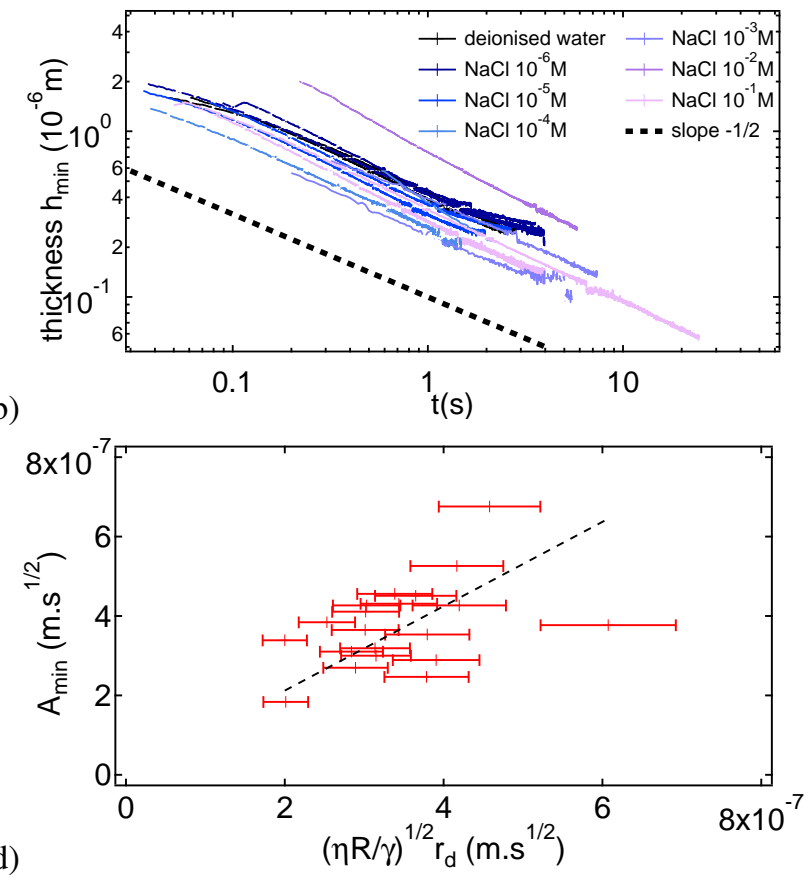

Fig. 7 Capillary regime of drainage: dynamics of $h_{0}$ (a) and $h_{\min }$ (b) for a series of $\mathrm{NaCl}$ concentrations (Black: deionized water, and from darker to lighter $\left.[\mathrm{NaCl}]=10^{-6}, 10^{-5}, 10^{-4}, 10^{-3}, 10^{-2}, 10^{-1} \mathrm{M}\right)$ and varied dimple radii $r_{d}$ and drop radius $R$. Data for $t<t_{1}$ only is plotted here for clarity (a) Dashed line - power law with exponent -1/4. Fit of the data to power law $A_{0} t^{-\mu}$ gives $\mu=0.26 \pm 0.03$ (b) Dashed line power law with exponent -1/2. Fit of the data to power law $A_{\min } t^{-v}$ gives $v=0.55 \pm 0.04$ (c) Experimental prefactor $A_{0}$ versus prediction $\left(\frac{\eta r_{d}^{6}}{\gamma R}\right)^{1 / 4}$ from Eq. 13. (d) Experimental prefactor $A_{\min }$ versus prediction $\sqrt{\frac{\eta R r_{d}^{2}}{\gamma}}$ from Eq. 14. (c,d) Dashed line: fit to a line. 


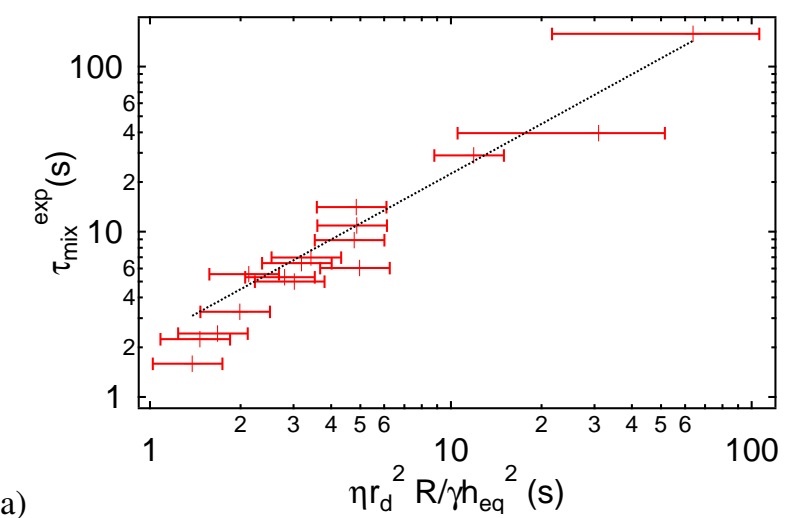

(a)

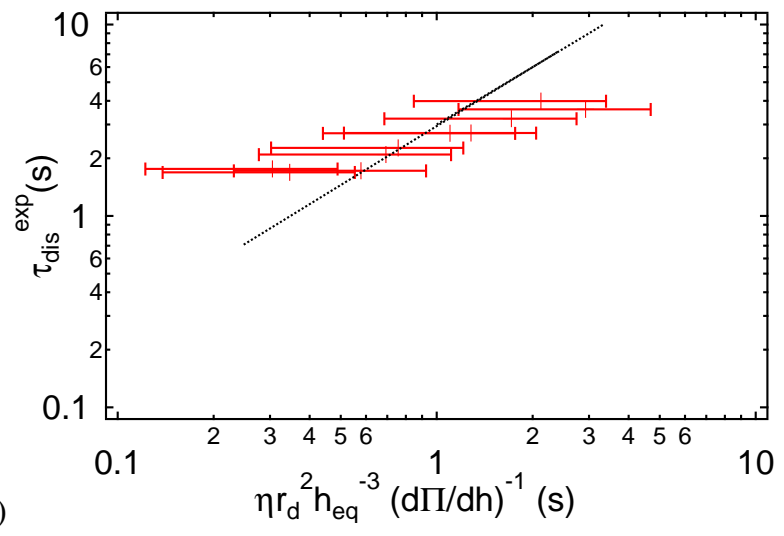

Fig. 8 Mixed and disjoining pressure regimes of the drainage: characteristic times of drainage for deionised water and $\mathrm{NaCl}$ solutions of concentration $10^{-6}, 10^{-5}, 10^{-4} \mathrm{M}$ in (a) Mixed regime $\left(\tau_{m i x}\right)$ as a function of theoretical prediction (Eq. 19) and (b) Disjoining pressure regime $\left(\tau_{d i s}\right)$ as a function of theoretical prediction (Eq. 20). Dotted lines: fit to a line with numerical prefactor $\alpha$ as adjustable parameter: we find (a) $\alpha_{\text {mix }}=2.2 \pm 0.1$, (b) $\alpha_{d i s j}=3 \pm 1$.

allow to derive simple scaling laws presented in the Appendix, thus providing a relation between the thickness at the center $h_{0}$ and its time derivative:

$$
\frac{2 \gamma}{R}=\frac{9 \eta r_{d}^{2}}{2 h_{e q}^{2}} \frac{\dot{h_{0}}}{h_{0}}
$$

Within this approximation, the thickness at the center relaxes exponentially over time with a characteristic time equal to:

$$
\tau_{m i x}=\frac{9}{4} \frac{\eta r_{d}^{2} R}{\gamma h_{e q}^{2}}
$$

The exponential decay is indeed observed experimentally as shown in Fig. 2c). The characteristic time of this decay was measured for a series of experiments with varied salt concentration and drop radii of curvature $R$. In Fig. 8a) the results are reported as a log-log plot as a function of the theoretical factor $\left(\frac{\eta r_{d}^{2} R}{\gamma h_{e q}^{2}}\right)$ without numerical prefactor. The data can be fit to a line with slope 1 to a very good approximation. Moreover the numerical prefactor we deduced is $2.2 \pm 0.1$, and is also in good agreement with the theoretical one of 9/4. The quantitative agreement between our model and our experiments confirms that we have a quantitative understanding of this mixed regime. The exponential dependence of the drainage and the qualitative impact of the electrolyte concentration on its characteristic time were evidenced in the past. ${ }^{12}$ However, the time scales observed by Goodall et al. are about a hundred times larger than ours. This huge difference must be due to a difference in the geometrical lengths $r_{d}$ and $R$. Indeed, our model evidences the critical role played by those lengths in the exponential decay (see Eq. 19). Because the values of $r_{d}$ and $R$ are not clear in the cited paper, we cannot compare their time scales with the previsions of our theoretical model. Besides, Goodall et al. conclude from their experiments that the drainage rate is higher with the decrease of the ionic strength. However, our model predicts the inverse trend: as $h_{e q}$ is decreasing with the salt concentration, the higher the concentration of the salt, the longer the drainage as observed experimentally in the present study. This trend is also supported by Hewitt et al. ${ }^{25}$ Altogether, our theoretical model along with our experimental results evidence the critical role of the dimple geometry and clarify the impact of salt on the drainage rate.

\subsection{Disjoining pressure regime}

As the thickness at the dimple center thins even more, the drainage enters the third regime in which the full film lays within the range of disjoining pressure effects. In that case, as detailed in the Appendix, and verified experimentally from the measurement of the crossover thickness $h_{2}$, the pressure gradient between the center and the edge of the dimple is dominated by the disjoining pressure gradient. We find that the thickness at the dimple center $h_{0}$ relaxes exponentially again, with a characteristic time $\tau_{d i s}$ given by:

$$
\tau_{d i s}=2,08 \frac{\eta r_{d}^{2}}{h_{e q}^{3}}\left(-\left.\frac{\partial \Pi}{\partial h}\right|_{h_{e q}}\right)^{-1}
$$

Our data in the third regime were successfully fit to an exponentially decaying function of time and the characteristic time is plotted in Fig. 8b) as a function of the theoretical factor $\frac{\eta r_{d}^{2}}{h_{e q}^{3}}\left(-\left.\frac{\partial \Pi}{\partial h}\right|_{h e q}\right)^{-1}$ where the disjoining pressure dependent quantity was computed from the fit in Fig. 4. The data are quite noisy, and this is likely due to the irreproducibility of the disjoining pressure while renewing the solid and liquid interfaces. Indeed, the potential $\psi$ of the glass interface was 
found to vary experimentally and could not be determined for all drainage experiments. However the order of magnitude is extremely good. The linear fit to the data provides a numerical factor of $3 \pm 1$ while the theorical prediction is 2.08 . The agreement between our model and our observations confirms here the relevance of our analysis of this disjoining pressure regime.

\section{Conclusion}

In this paper, we present the drainage of a water thin film between an oil drop and a borosilicate glass focusing on the time evolution of the thicknesses at the center $h_{0}$ and at the barrier ring $h_{\text {min }}$, both experimentally and theoretically. We experimentally highlight three different regimes of drainage. We model the first two regimes by considering that the viscous dissipation is located at the barrier ring. The first regime is driven by the oil/brine interfacial tension and exhibits a power law dependence of both thicknesses with time. The second one presents a barrier ring thickness controlled by the disjoining pressure. The modeling of the third one is based on a pressure gradient across the film described by the disjoining pressure only. The second and third regimes exhibit drainage dynamics with thicknesses exponentially decaying over time with a characteristic time we predict.

The regime changes correspond to thicknesses $h_{\text {min }}$ or $h_{0}$, depending on the considered regimes, becoming small enough for disjoining pressure effects to become non negligible compared to the capillary pressure at the barrier ring or at the center respectively.

This approach allows for the derivation of simple laws for thickness variations in the three regimes. The scaling laws obtained in the first regime are in agreement with previous works. The variation laws in the two subsequent regimes, in which disjoining pressure effects are at stake, have not been established before. Our experimental data is very good agreement with our models. Since the simple laws we provide do not assume any specific form for the disjoining pressure, they constitute a new and general framework to analyse the drainage dynamics of a liquid film squeezed between a solid surface and either a drop or a bubble. In particular, they allow for the decoupling of capillary and disjoining pressure effects from geometrical effects and should help to improve the general understanding of the phenomena at stake.

\section{Appendix}

With typical thickness $h \sim 1 \mu \mathrm{m}$ and diameter $2 r_{d} \sim 100 \mu \mathrm{m}$, the aspect ratio of the film $h / r_{d}$ is of order $10^{2}$ and the lubrication approximation holds. The lubrication assumes that the radial velocity has a parabolic profile. And the velocity

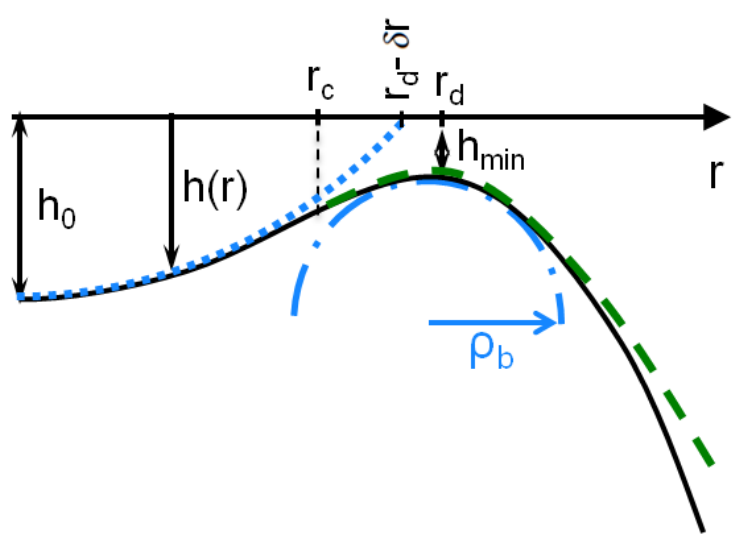

Fig. 9 Geometrical parameters characterizing the radial film thickness profiles $h(r): h_{0}$ : thickness at the center; $h_{\min }$ : minimum thickness at the barrier ring, which corresponds to a radial distance $r_{d}$; the dimple center area is fit to a second order polynomial $\left(h_{d}\right.$, Eq. 27, dotted line); and the barrier ring to a third order polynomial $\left(h_{b}\right.$, Eq. 26, dashed line) with radius of curvature $\rho$ (dash-dotted line). The two polynomials connect at $r=r_{c}$.

vanishes at the solid interface, at $z=0$. The velocity profile writes:

$$
v_{r}=\frac{6 q}{3 \beta-2} \frac{z(\beta h-z)}{h^{3}}
$$

where $q$ is the radial water flux per unit of perimeter and $\beta$ is a parameter related to boundary condition at the liquid/liquid interface: if the tangential stress vanishes at this interface, $\beta=$ 2 ; if the tangential velocity cancels, $\beta=1$. Thus in the frame of the lubrication approximation, the Stokes equation (Eq. 1) combined with the velocity profile (Eq. 21 ) reduces to:

$$
\frac{\partial P}{\partial r}=-\eta \frac{12 q}{(3 \beta-2) h^{3}}
$$

The pressure can be written as the sum of the opposite of the disjoining pressure $\Pi$ and the capillary pressure, for which the oil/water interface curvature can be written from $h$ and its radial derivatives. The general expression of the pressure gives:

$$
P=P_{e x t}+2 \gamma R^{-1}-\gamma\left(\frac{\partial^{2} h}{\partial r^{2}}+\frac{1}{r} \frac{\partial h}{\partial r}\right)-\Pi
$$

Finally, the flux $q$ depends on $r$ and is related to the motion of the liquid/liquid interface by the volume conservation equation:

$$
\frac{\partial r q}{\partial r}=-r \frac{\partial h}{\partial t}
$$

The combination of Eqs. 22, 23 and 24 gives the governing equation (Eq. 2) for the space and time variations of the film thickness $h$, that cannot however be solved analytically. We 
present here a novel scaling analysis that allows to capture the main features of the dimple relaxation. We will assume for the sake of simplicity that $\beta=1$ as observed experimentally, but the general case can be easily derived.

\subsection{Capillary driven regime}

We assume that the barrier ring is immobile at a radial distance $r_{d}$ and that the pressure gradient is dominated by the interfacial tension term: the gradient of disjoining pressure is negligible. Finally the curvature of the interface simply writes $\frac{\partial^{2} h}{\partial r^{2}}$ because the problem is quasi one-dimensional. Eq. 2 reduces to:

$$
\frac{12 q_{b} \eta}{h_{\min }^{3}}=\left.\gamma \frac{\partial^{3} h}{\partial r^{3}}\right|_{r_{d}}
$$

where $q_{b}$ is the flux at the barrier ring located at radius $r_{d}$. At the barrier ring, the height $h$ presents a minimum, and can be expanded in terms of $r-r_{d}$. This expansion has to include a third order term, because Eq. 25 contains a third order derivative of $h$. Indeed, taking into account this third order terms is the main originality of our present calculation. To the third order, the shape of the barrier ring writes, for $r$ close to $r_{d}$ :

$$
h_{b}(r) \simeq h_{\min }+\frac{\left(r-r_{d}\right)^{2}}{2 \rho_{b}}+\frac{\left(r-r_{d}\right)^{3}}{6 G^{2}}
$$

where $\rho_{b}$ is the radius of curvature at the barrier ring and $G$ is a parameter related to the barrier ring asymmetry. The barrier ring shape has to be connected to the dimple center. At the center, the dimple shape is approximatively parabolic and thus writes:

$$
h_{d}(r) \simeq h_{0}\left(1-\frac{r^{2}}{\left(r_{d}-\delta r\right)^{2}}\right)
$$

where $\left(r_{d}-\delta r\right)$ is defined in Fig. 9 as the radius for which the parabolic shape $h_{d}$ is null. To connect the center $h_{d}$ and the barrier ring $h_{b}$, we write that at a given value of $r$, that we will call $r_{c}$, the height $h$ as well as its first and second derivatives are continuous, i.e. $h_{d}\left(r_{c}\right)=h_{b}\left(r_{c}\right), h_{d}^{\prime}\left(r_{c}\right)=h_{b}^{\prime}\left(r_{c}\right)$ and $h_{d}^{\prime \prime}\left(r_{c}\right)=h_{b}^{\prime \prime}\left(r_{c}\right)$. These three equations lead after some calculations, and in the limit where $\delta r \ll r_{d}$, and $h_{0} \ll r_{d}$, to the following geometrical relations:

$$
\begin{aligned}
G & \simeq 2 \rho_{b} \sqrt{\frac{h_{0}}{r_{d}}} \\
\rho_{b} & \simeq \frac{3}{8} \frac{h_{\min r_{d}^{2}}}{h_{0}^{2}} \\
r_{d}-r_{c} \simeq \frac{G^{2}}{\rho_{b}} & \simeq \frac{3}{2} \frac{h_{\min } r_{d}}{h_{0}}
\end{aligned}
$$

Our model equations (Eqs. 26 and 27) of the film geometry were successfully fit to actual film profiles (see Fig. 5a), showing the relevance of our description. Futhermore, the adjustable parameter $G, \rho_{b}$ and $r_{c}$ obtained from these fits were found to compare well with the numerical evaluations of Eqs. 28, 29, 30 using our measurements of the other relevant lengths $\left(h_{0}, h_{\min }, r_{d}\right)$. This validates the present description.

Combining Eqs. 25 and 26, we obtain:

$$
\frac{12 q_{b} \eta}{h_{\min }^{3}}=\frac{\gamma}{G^{2}}
$$

Combined with Eq. 30, this sets all the geometrical parameters for a given value of the flux. From Eqs. 28, 29, 31, we can eliminate $G$ and $\rho_{b}$ and get a relation between the gap $h_{\text {min }}$ and the flux $q_{b}$ at the barrier ring:

$$
h_{\text {min }}=\frac{27 q_{b} \eta r_{d}^{3}}{4 \gamma h_{0}^{3}}
$$

Now, the flux and the geometry can be related in two ways. One originates simply from volume conservation while the second is related to the pressure drop between the dimple and the reservoir. Assuming the dimple center remains parabolic, and the radius at the barrier ring remains constant lead to the following relation in the dimple:

$$
q(r)+\frac{\dot{h_{0}} r}{2}\left(1-\frac{1}{2}\left(\frac{r}{r_{d}}\right)^{2}\right) \simeq 0
$$

where $\dot{h_{0}}$ is the time derivative of $h_{0}(t)$. The flux at the barrier ring can therefore be estimated by:

$$
q_{b} \cong-\frac{\dot{h_{0}} r_{d}}{4}
$$

The pressure drop across the dimple, which is equal to the difference between the capillary pressure in and out of the dimple, can be approximated in the capillary regime to $2 \gamma / R$. This pressure drop is given by the integration over $r$ of Eq. 22 that will be next approximated. Most of the pressure drop is localized within the barrier ring, so that:

$$
\Delta P=P(0)-P_{e x t}=\int_{0}^{\infty} \eta \frac{12 q(r)}{h(r)^{3}} d r \gtrsim \int_{r_{c}}^{r_{d}} \eta \frac{12 q_{b}}{h_{b}(r)^{3}} d r
$$

One also have to note that the barrier ring is strongly asymmetric as emphasized by the third order term of $h_{b}(r)$ in $\left(r-r_{d}\right)^{3} / 6 G^{\prime 2}$ (see Eq. 26). This term actually dominates the pressure drop $\Delta P$. Indeed one can note that $h\left(r_{c}\right) \cong 3 h_{\text {min }}$. Thus the pressure drop is asymmetric at the barrier ring and localized mostly between the connection to the parabolic dimple $r_{c}$ and the barrier ring itself at $r_{d}$ as can be seen in Fig. 5b). We 
will simply assume that the film shape is nearly flat between $r_{c}$ and $r_{d}$, and that the integral 35 can be estimated to:

$$
\Delta P \cong \frac{12 q_{b} \eta\left(r_{d}-r_{c}\right)}{h_{\min }^{3}}
$$

Indeed the two approximations - the one of Eq. 35, and the approximation of a flat barrier ring between $r_{c}$ and $r_{d}$ - respectively underestimate and overestimate the pressure drop. As a result, the approximation is indeed very good. As explained in the article body, our model gives satisfactory descriptions of the whole phenomenon.

Combining the previous expressions, we recover the two relations for the time dependence of $h_{0}$ and $h_{\min }$ found in the literature, ${ }^{3,7}$ with different prefactors:

$$
\begin{aligned}
h_{0}(t) & \simeq \frac{3}{4}\left(\frac{\eta r_{d}^{6}}{\gamma R}\right)^{1 / 4} t^{-1 / 4} \\
h_{\min }(t) & \simeq \frac{3}{4} \sqrt{\frac{\eta R r_{d}^{2}}{\gamma}} t^{-1 / 2}
\end{aligned}
$$

Thus thanks to our analysis, we are able to recover these scaling laws given by other means. This allows us to address the second regime, where the disjoining pressure acts on the barrier ring but not on the dimple.

\subsection{Mixed regime}

In the mixed regime, the barrier ring height $h_{\min }$ has nearly its asymptotic value $h_{e q}$ : indeed, experimentally, in regime (ii), $h_{\min }$ varies between twice $h_{e q}$ and $h_{e q}$ as exemplified in Fig. 2. The thickness at the barrier ring is therefore set by the disjoining pressure. On the other hand, the film drainage is set by the pressure gradient, so that we shall compare the relative contributions to the pressure gradient of the disjoining and capillary contributions in Eq. 23. Similarly to the derivation of Eq. 25, the capillary term writes $\gamma \frac{\partial^{3} h}{\partial r^{3}}$ and provides a zero order term in $r-r_{d}$ to the pressure gradient. The disjoining pressure gradient writes: $\frac{\partial \Pi}{\partial r}=\frac{d \Pi}{d h} \frac{\partial h}{\partial r}$. As the barrier ring corresponds to a minimum of the thickness profile, the expansion of $h$ about $h_{\min }$ (Eq. 26) has no first order term and the disjoining pressure gradient is therefore a higher order term in $r-r_{d}$. As such, the disjoining pressure gradient across the barrier ring is negligible compared to the capillary pressure gradient. Finally, the capillary pressure gradient must be compared to the term $2 \gamma R^{-1}$. Experimentally, the latter is always dominating the former. Therefore, the pressure gradient across the barrier ring of length $\left(r_{c}-r_{c}\right)$ writes: $2 \gamma R^{-1}\left(r_{d}-r_{c}\right)^{-1}$. From Eq. 22, the flux at the barrier ring $q_{b}$ can be derived:

$$
\frac{2 \gamma}{R\left(r_{d}-r_{c}\right)}=\frac{12 q_{b} \eta}{h_{e q}^{3}}
$$

The main difference with the capillary driven regime (i) lays in the height at the barrier ring being set by disjoining pressure rather that the viscous lift. Expliciting the flux from Eq. 34, and $r_{d}-r_{c}$ from Eq. 30, we obtain:

$$
\frac{2 \gamma}{R}=-\frac{9 \eta r_{d}^{2}}{2 h_{e q}^{2}} \frac{\dot{h_{0}}}{h_{0}}
$$

The relaxation of the dimple center of thickness $h_{0}(t)$ is exponential, with a relaxation time equal to:

$$
\tau_{m i x}=\frac{9 \eta r_{d}^{2} R}{4 \gamma h_{e q}^{2}}
$$

\subsection{Disjoining pressure regime}

For this regime, the film is not far from equilibrium for which $P(r)=P_{\text {ext }}$. Observing that the film is almost flat, we can assume that the capillary pressure gradient is negligible compared to the disjoining pressure gradient across the film. From Eqs. 22, 23, 24:

$$
\frac{\partial h}{\partial t}+\frac{1}{r} \frac{\partial}{\partial r}\left(r \frac{1}{12} \frac{h^{3}}{\eta} \frac{\partial \Pi}{\partial r}\right)=0
$$

Linearizing this equation about $h=h_{e q}$, leads to:

$$
\dot{h}+\left(\left.\frac{1}{12} \frac{h_{e q}^{3}}{\eta} \frac{\partial \Pi}{\partial h}\right|_{h_{e q}}\right) \frac{1}{r} \frac{\partial}{\partial r} r \frac{\partial h}{\partial r}=0
$$

The solution of this equation can be written as $h=h_{e q}+$ $\sum a_{\tau, k} e^{-\frac{t}{\tau}} J_{0}(k r)$ where $J_{0}$ is the Bessel function, $a_{\tau, k}$ is the amplitude of the $(\tau, k)$ mode for which the relation between $\tau$ and $k$ is given by:

$$
-\left(\left.\frac{1}{12} \frac{h_{e q}^{3}}{\eta} \frac{\partial \Pi}{\partial h}\right|_{h_{e q}}\right) k^{2} \tau=1
$$

In this last regime, $h_{\min }$ at the barrier ring is set to $h_{e q}$. In other words, the profiles are pinned to the boundary condition: $h\left(r=r_{d}\right)=h_{e q}$ so that $k$ obeys $J_{0}\left(k r_{d}\right)=0$. Therefore, the slowest mode is given by the first zero of the Bessel function i.e. for $k r_{d}=2.40$. Finally, the film profile $h(r, t)$ relaxes to the equilibrium thickness $h_{e q}$ within a characteristic time $\tau_{d i s}$ given by:

$$
\tau_{d i s} \sim-\frac{12}{(2.40)^{2}} \frac{\eta r_{d}^{2}}{h_{e q}^{3}}\left(\left.\frac{\partial \Pi}{\partial h}\right|_{h_{e q}}\right)^{-1}
$$




\section{References}

1 D. Y. C. Chan, E. Klaseboer and R. Manica, Soft Matter, 2011, 7, 22352264.

2 D. G. Goodall, M. L. Gee and G. W. Stevens, Langmuir, 2002, 18, 47294735.

3 S. P. Frankel and K. J. Mysels, The Journal of Physical Chemistry, 1962, 66, 190-191.

4 R. K. Jain and I. B. Ivanov, Journal of the Chemical Society, Faraday Transactions 2: Molecular and Chemical Physics, 1980, 76, 250-266.

5 J. N. Connor and R. G. Horn, Faraday Discussions, 2003, 123, 193-206.

6 R. Manica, J. N. Connor, L. Y. Clasohm, S. L. Carnie, R. G. Horn and D. Y. C. Chan, Langmuir, 2008, 24, 1381-1390.

7 S. Hartland and J. D. Robinson, Journal of Colloid and Interface Science, 1977, 60, 72-81.

8 D. S. Dimitrov and I. B. Ivanov, Journal of Colloid and Interface Science, 1978, 64, 97-106.

9 S. I. Karakashev, K. W. Stockelhuber, R. Tsekov and G. Heinrich, Journal of Colloid and Interface Science, 2013, 412, 89-94.

10 C. S. Tan, M. L. Gee and G. W. Stevens, Langmuir, 2003, 19, 7911-7918.

11 D. G. Goodall, M. L. Gee and G. W. Stevens, Langmuir, 2001, 17, 3784 3787.

12 D. G. Goodall, G. W. Stevens, D. Beaglehole and M. L. Gee, Langmuir, 1999, 15, 4579-4583.

13 R. G. Horn, M. Asadullah and J. N. Connor, Langmuir, 2006, 22, 26102619.

14 J. N. Connor and R. G. Horn, Langmuir, 2001, 17, 7194-7197.

15 R. Manica and D. Y. C. Chan, Phys. Chem. Chem. Phys., 2011, 13, 1434 1439.

16 E. Verneuil, J. Clain, A. Buguin and F. Brochard-Wyart, The European Physical Journal E - Soft Matter, 2003, 10, 345-353.

17 J. Blass, O. Kohler, M. Fingerle, C. Muller and C. Ziegler, Physica Status Solidi (a), 2013, 210, 988-993.

18 A. C. Lam and R. S. Schechter, Journal of colloid and interface science, 1991, 146, 206-214.

19 T. D. Dimitrova, F. Leal-Calderon, T. D. Gurkov and B. Campbell, Langmuir, 2001, 17, 8069-8077.

20 Stubenrauch, Cosima and von Klitzing, Regine, J. Phys.: Condes. Matter, 2003, 15, R1197-R1232.

21 K. G. Marinova, R. G. Alargova, N. D. Denkov, O. D. Velev, D. N. Petsev, I. B. Ivanov and R. P. Borwankar, Langmuir, 1996, 12, 2045-2051.

22 R. Zimmermann, U. Freudenberg, R. Schweiss, D. Kuttner and C. Werner, Current Opinion in Colloid \& Interface Science, 2010, 15, 196-202.

$23 \mathrm{~K}$. Roger and B. Cabane, Angewandte Chemie International Edition, 2012, 51, 5625-5628.

24 Y. Gu and D. Li, Journal of Colloid and Interface Science, 2000, 226, 328-339.

25 D. Hewitt, D. Fornasiero, J. Ralston and L. R. Fisher, Journal of the Chemical Society, Faraday Transactions, 1993, 89, 817-822.

26 Israelachvili, J., Intermolecular and Surface Forces, Academic Press, San Diego (USA), 3rd edn, 2011.

27 O. Manor, I. U. Vakarelski, X. Tang, S. J. O Shea, G. W. Stevens, F. Grieser, R. R. Dagastine and D. Y. C. Chan, Physical Review Letters, 2008, 101, year.

28 L. Y. Clasohm, J. N. Connor, O. I. Vinogradova and R. G. Horn, Langmuir, 2005, 21, 8243-8249.

29 Klaseboer, E., Chevaillier, J.P., Gourdon, C. and Masbernat, O., Journal of Colloid and Interface Science, 2000, 229, 274-285.

30 Probstein Ronald F., Physicochemical Hydrodynamics: An Introduction, Wiley, Hoboken (New Jersey), 2nd edn, 2003. 